\title{
Distributed power control with received power constraints for time-area-spectrum licenses is
}

\author{
Ana Pérez-Neira ${ }^{\mathrm{a}, \mathrm{b}, *, 1}$, Joaquim M. Veciana ${ }^{\mathrm{c}}$, Miguel Ángel Vázquez ${ }^{\mathrm{a}}$, \\ Eva Lagunas ${ }^{\mathrm{d}, 2}$ \\ a Centre Tecnològic de Telecomunicacions de Catalunya (CTTC), Av. Carl Friedrich Gauss 7, 08860 Castelldefels, Spain \\ ${ }^{\mathrm{b}}$ Department of Signal Theory and Communications, Universitat Politècnica de Catalunya (UPC), C. Jordi Girona 1-3, 08034 Barcelona, \\ Spain \\ ${ }^{\mathrm{c}}$ Department of Mechanical Engineering, Universitat Politècnica de Catalunya (UPC), Av. Diagonal 647, 08028 Barcelona, Spain \\ d Interdisciplinary Centre for Security, Reliability and Trust (SnT), University of Luxembourg, rue Alphonse Weicker 4, L-2721, Luxembourg
}

\section{A R T I C L E I N F O}

\section{Article history:}

Received 2 March 2015

Received in revised form

22 July 2015

Accepted 4 September 2015

Available online 25 September 2015

\section{Keywords:}

Power control

Spectrum sharing

Interference channel

Spectrum license

\begin{abstract}
A B S T R A C T
This paper deals with the problem of optimal decentralized power control in systems whose spectrum is regulated in time and space, the so-called time-area-spectrum (TAS) licensed. In this paper we consider those locations with colliding transmissions; thus, addressing a scenario with full interference. In order to facilitate the coexistence of different TAS licenses, the power spectral density of the used band shall be limited. Since controlling the overall radiated power in a given area is cumbersome, we control the amount of received power. First, we present the achievable rates (i.e. the rate Pareto set) and their corresponding powers by means of multi-criteria optimization theory. Second, we study a completely decentralized and gradient-based power control that obtains Pareto-efficient rates and powers, the so-called DPC-TAS (Decentralized Power Control for TAS). The power control convergence and the possibility of guaranteeing a minimum Quality of Service (QoS) per user are analyzed. Third, in order to gain more insight into the features of DPC-TAS, this paper compares it with other baseline power control approaches. For the sake of comparison, a simple pricing mechanism is proposed. Numerical simulations verify the good performance of DPC-TAS.
\end{abstract}

(c) 2015 Elsevier B.V. All rights reserved.

\footnotetext{
The work by A. Pérez-Neira and M.A. Vázquez was supported by the Spanish Ministry of Economy and Competitiveness (Ministerio de Economia y Competitividad) under project TEC2014-59255-C3-1-R, by the Catalan Government under grant 2014SGR1567 and by the European Commission through the SANSA project (ICT-645047). The work by E. Lagunas was supported by the National Research Fund, Luxembourg, under CORE project SpEctrum Management and Interference mitiGation in cognitive raDio satellite networks - SeMIGod and by the European Commission through the SANSA project (ICT-645047). Part of this work has been published in IEEE Applied Electromagnetics Conference (AEMC), Kolkata, India (December 2011).

* Corresponding author at: Department of Signal Theory and Communications, Universitat Politècnica de Catalunya (UPC), C. Jordi Girona 1-3, 08034 Barcelona, Spain. Tel.: +3493 4016459; fax: +34 934016440.

E-mail addresses: ana.isabel.perez@upc.edu (A. Pérez-Neira), joaquim.maria.veciana@upc.edu (J.M. Veciana), mavazquez@cttc.es (M.Á. Vázquez), eva.lagunas@uni.lu (E. Lagunas).

${ }_{1}^{1}$ Member of EURASIP (No. 5934).

${ }^{2}$ Member of EURASIP (No. 7784).
} 


\section{Introduction}

Wireless technology is proliferating rapidly requiring more radio spectrum. In light of this, spectrum sharing has gained a special attention in the research community for its promising results in improving the spectral efficiency. The concept of Cognitive Radio (CR) [1-3] has been hailed as a potential communication paradigm, which allows low-priority systems to sense their operating environment and adapt their implementation to achieve the best performance while minimizing harmful interference to other users. While the concept of $\mathrm{CR}$ networks has been well accepted within the wireless communications research community, potential benefactors and regulation authorities have shown strong reluctance to the application of CR in real world scenarios [4]. There are two major hurdles for CR networks to come true. First, a multiple secondary user environment in which the number of cognitive devices is large, might lead to a spectrum saturation and might cause severe interference to the incumbent system. Second, the efficiency and reliability of present spectrum sensing techniques to predict the performance of the primary communication link is often questionable and the time spent in acquiring this information is also one of the main concerns.

An early attempt to overcome such limitations is the Authorized/Licensed Shared Access (ASA/LSA) approach [5], which provides new sharing opportunities under a licensing regime. LSA provides a means for incumbent spectrum holders to make available, subject to sharing and commercial agreement, their spectrum for wireless services. LSA has shown great promise in making spectrum sharing attractive for mobile operators. One possible system architecture for LSA is the time-area-space (TAS) licenses [6,7]. TAS license concept was first introduced in [6] and it provides a more efficient spectrum management system than the current open spectrum one. The reason is that this regulation technique not only controls frequency, but also time and location. In other words, whenever a certain number of users (operators) acquire a TAS license, the spectrum regulator assigns to these incumbents the right of transmitting in a given frequency for a certain portion of time within some geographical limits.

In order to allow the creation of geographically close TAS licenses, the power spectral density within the TAS license area shall be restricted. Unfortunately, controlling the radiated power in a given area is cumbersome, due to the stochastic nature of the radio channel and to correlations among transmitting antennas (i.e. when there are more than one). Furthermore, these spatial-frequency restrictions can only be managed by a central controller, which would require a large amount of signalling among the different communication agents.

An alternative to restricting the radiated power in a given area is to limit the total amount of received power [8]. With this, the received power constraints can approximate the spatial interference power restrictions, leading to a more flexible management of the power and, ultimately, of the license, as we show in the present paper. Fig. 1 illustrates a possible scenario, where, by guarantee

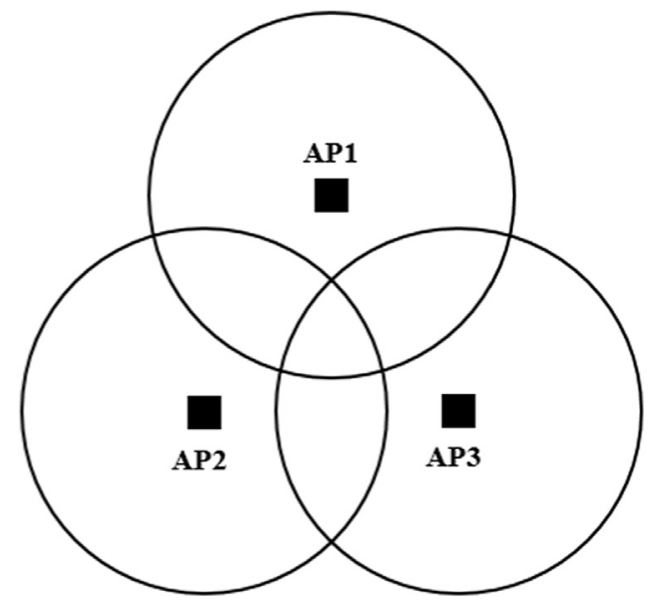

Fig. 1. Scenario with 3 access points operating on the same frequency band but in spatial mostly disjoint areas. There is no cooperation at any level between the 3 systems; circles indicate the coverage due to constraints on the maximum transmit power.

on the maximum level of received signal, this paper solves how to enable coexistence in the overlapping areas.

In contrast to other spectrum regulations, which restrict the power density in a per-user basis (e.g. maximum radiated power and maximum interference level to the primary user), TAS spectrum license grants the use of the spectrum on a network level fashion. This constitutes a substantial difference since all TAS incumbents shall coordinate in order to preserve the received power constraints. Indeed, this network-wide power restriction fosters the spectrum sharing among the TAS incumbents and it allows the coexistence of geographically adjacent TAS licenses since the overall spectral power density is approximately restricted with the receive power constraints.

Under this context, all users have the same privileges and they have to coordinate in order not to exceed the received power constraint. Note that it is a total received power constraint, which differs from the interference temperature constraint that is considered when the spectrum policy differentiates between primary and secondary users, as it is the case, for instance, in $[9,10]$. Finally, to understand Fig. 1, in addition to the received power constraint, there is always a constraint on the maximum transmit power; thus, conforming a coverage area around each access point.

\subsection{Related works and contributions}

In any receiver, Automatic Gain Control (AGC) tries to keep the received power at some nominal level by inverting the pathloss and fading effects of the channel. However, inverting the channel results in a capacity penalty. In order to obtain optimal power adaptation in terms of capacity waterfilling in time has to be implemented, analogous to waterfilling in frequency (see [11] and references therein); thus, requiring transmit, instead of receive, power control. When, in addition, the transmission is degraded by interference, the large dynamic range of signals that must be handled by most receivers requires a 
new gain adjustment. Due to the interference, this adjustment becomes complex as it has to deal with nonconvex utilities and, moreover, in the case of ad-hoc networks, it has to be implemented autonomously at each communication link.

Power control for interference management has been successfully applied in general communication systems [12-15]. The standard interference functions, which were introduced by Yates [16] and further studied and developed by Boche and Schubert [17], have been very influential on the analysis and design of distributed power control laws. The starting point for much of the research done in decentralized power control can be found in [18], where the authors propose an algorithm to meet Signalto-Interference-and-Noise-Ratio (SINR) requirements at each receiver with minimum power. This algorithm carries out fix point iterations [19], which can be speed up by applying the Perron-Frobenius (PF) duality [20]. From a different perspective, Chiang [21] proposes Geometric Programming (GP) to overcome the non-convexity barriers that wireless utility optimization problems present in interference limited scenarios. These works also obtain decentralized power control mechanisms. Of particular interest for the present work are [22] and [23], where a Lagrangian approach is taken together with gradientbased techniques. Specifically, Gatsis and Giannakis [24] discuss about the change of variables that are carried out in GP in order to convexify many power control problems. As a summary, in [25,26], the reader can find good reviews on power control in actual wireless networks.

One class of interference channel is cognitive radio for spectrum sharing, where users are classified as secondary/ unlicensed or primary/licensed users. Decentralized interference management and power allocation for both kinds of users have to be implemented with their corresponding different interference constraints [27-29]. In [30,31] the authors study the rate region frontiers in the interference channel when interference is treated as noise and there are constraints on the per-transmitter maximum available power. Borrowed from economic theory, game theory and pricing techniques have been widely studied in the recent years within this spectrum sharing context [32-37]. The reason is that game theory is a mathematical framework that focuses on how groups of people/users interact; thus, incorporating the required additional flexibility to obtain new power control policies within the spectrum sharing context. Some popular algorithms with interference pricing are $[38,39]$, where interference prices are announced to the network to reach an agreement among the communication nodes. Another interesting use of prices is to punish users' misbehavior as it is studied in [40].

In this paper, a Decentralized Power Control is proposed for the TAS spectrum sharing architecture, namely DPC-TAS and it is studied under a signal processing perspective. To the best of the authors knowledge, the first study of the TAS licenses, from the communication perspective, was the work by Gastpar in [41], which was later extended to relay networks in [42]. This pioneering work deeply studies how the capacity and the architecture of the system is modified when not only the transmit power constraints are considered, but also the received ones; in other words, the constraints are placed on the channel output signal. The present paper applies these received power constraints in an interference network, where a decentralized power control is needed. Specifically, this work extends previous authors publications [43], where we first introduced the autonomous power control for such network. Some recent works in [44-46] also consider a similar problem (i.e. with transmit sum-power constraint or with interference temperature constraint). In [44] game-theoretic tools are used to analyze games played by resource-constrained players. The authors provide a general framework. Interestingly, in [45] equilibrium pricing of interference in cognitive networks is studied, where prices are indicators of the spectrum congestion and are broadcast to the network to achieve convergence. Finally, in [46] all transmitters in the interference network are considered to be connected to a common energy source and optimal power control is developed for sum-rate maximization. Differently to these works, this paper studies a decentralized power control that does not require price broadcasting and takes into account total received power constraints. Very interesting is the work in [24], where several power control formulations for spectrum sharing scenarios are presented in a unified way with various constraints that couple the power variables, received power constraint among them. The present work is also based on gradient-based iterations, but, being the taylored for specific TAS scenario, the general tools that are developed in [24], as for instance, projections or convexifications, are not needed. More specifically, the contributions of the present paper are summarized as follows:

- We analyze the Pareto rates and corresponding transmit powers of multiple access points when, due to the TAS license, they have restricted the amount of received power and not only the available power at transmission.

- We study a decentralized power control with transmit and received power constraints (i.e. DPC-TAS) and prove that it converges towards a Pareto power solution in TAS.

- We compare the achieved rates by DPC-TAS with those in a system whose goal is rate balancing or per-link SINR-QoS constraints. We show that in a decentralized and user competitive system it can be more efficient, in terms of rates, to guarantee maximum received power per user rather than to guarantee minimum QoS per user.

- We position DPC-TAS among other baseline approaches as the one by Foschini and Miljanic in [18] and also with respect to a basic pricing power control.

- We propose a decentralized pricing mechanism with similar complexity to DPC-TAS, and study its convergence. In spite of being intuitive, it is more difficult to tune than the proposed DPC-TAS, specially when the number of communication links increase.

- A thorough performance assessment is also given, and the results verify the good performance of the proposed DPC-TAS.

This work uses basic concepts as: gradient-based iterations, linear programming and pricing. The purpose 
is to clarify the relationship among them, with the ultimate goal of presenting and reinforcing a simple decentralized power control for the interference channel with TAS regulation.

The rest of the paper is organized as follows. Section 2 provides the problem statement and insights on the received power restrictions. In Section 3, the optimal policy for the transmit power control is identified and the achievable rates are derived. Section 4 studies the convergence of a TAS decentralized iterative power allocation method and discusses on the integration of minimum QoS requirements per user. In order to compare the DPC-TAS with other baseline alternatives, a simple pricing mechanism is proposed in Section 5. Section 6 shows the numerical simulations and Section 7 concludes the paper.

Notation: Throughout the paper, scalars are denoted by non-boldface type, vectors by boldface lowercase letters and matrices by boldface uppercase letters. Superscripts $(\cdot)^{\mathrm{T}}$ and $(\cdot)^{\mathrm{H}}$ denote transpose and complex conjugate transpose, respectively. Let $\preceq$ denotes the vector component-wise inequality.

\section{System model and problem statement}

We consider a scenario where $K$ neighbor access points transmit information to their intended receivers sharing frequency and time resources. The transmitted power of the $k$-th access point is $x_{k}$ and we define $\mathbf{x}=\left[\begin{array}{lll}x_{1} & \ldots & x_{\mathrm{K}}\end{array}\right]^{\mathrm{T}}$ as the power allocation vector. The link gain from the transmitter $i$ to receiver $j$ is denoted by $a_{i j}$. Matrix $\mathbf{A} \in \mathbb{R}^{\mathrm{K} \times \mathrm{K}}$ contains all the link gains of the network, $[\mathbf{A}]_{i j}=a_{i j}$. Moreover, the maximum transmitted power for the $k$-th access point is $p_{k}$ and we define $\mathbf{p}=\left[\begin{array}{lll}p_{1} & \ldots & p_{\mathrm{K}}\end{array}\right]^{\mathrm{T}}$. A simplified TAS network with $K=2$ access points is shown in Fig. 2.

Regardless that throughout the paper we refer to the regulation mask in terms of power, this is for the sake of presentation, since in fact the regulation specifies the mask in terms of power spectral density $(\mathrm{W} / \mathrm{Hz})$. In this paper we focus on the design of transmit power control that takes into account the amount of received signal power, which is restricted to $p_{\text {reg }}$ and, without loss of

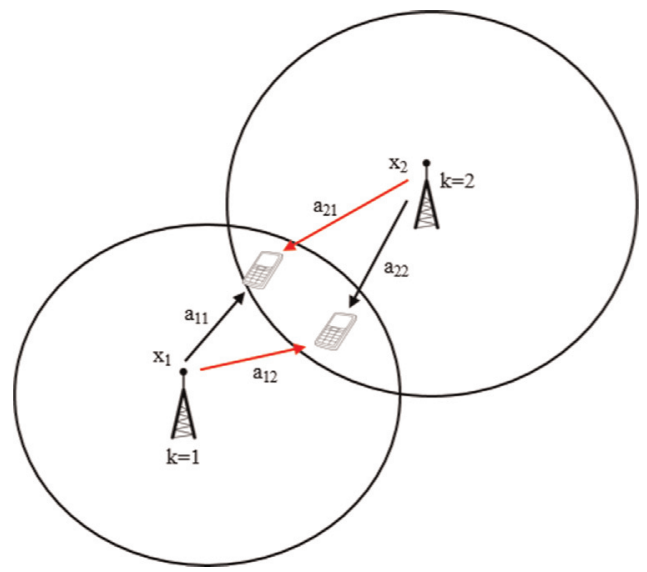

Fig. 2. Simplified scheme of TAS network with $K=2$ access points. generality, it is assumed the same for all link pairs. Note that with this constraint, coverage control is different from the circular areas, centered at each transmitter, that results when only maximum available transmit power is controlled (see Fig. 1). By limiting the received power the coverage area cannot be predicted as it is a point-wise constraint that is imposed on each of the participating receivers.

We aim to find all optimal rate pairs of this communication system, when the receivers implement single user detection and their received power is limited. Under that context, the achievable rate by user $k, k=1, \ldots, \quad \mathrm{K}$, is

$r_{k}=\log _{2}\left(1+\frac{a_{k k} x_{k}}{\sum_{\substack{j=1 \\ j \neq k}}^{K} a_{j k} x_{j}+\sigma^{2}}\right)=\log _{2}\left(1+\operatorname{SINR}_{k}\right)$.

where, without loss of generality, it has been assumed that the noise power level, $\sigma^{2}$, is equal for all receivers. We consider along the paper $\sigma^{2}=1$. Achieving all optimal rate points is defined as the solution of the following multicriteria optimization problem (MOP) $[47,48]$

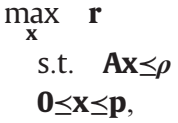

where $\mathbf{r}=\left[\begin{array}{lll}r_{1} & \ldots & r_{\mathrm{K}}\end{array}\right]^{\mathrm{T}}$ and $\boldsymbol{\rho}$ is a vector that includes the $\mathrm{K}$ regulatory constraints minus the noise power $[\rho]_{k}=p_{\text {reg }}-\sigma^{2}$. The term $p_{\text {reg }}$ refers to the maximum receive power imposed by the TAS spectrum regulation.

It is worth mentioning that the aforementioned optimization problem is described considering the notation that appears both in [47] and [49]. MOP is an area of optimization theory that is concerned with mathematical optimization problems involving more than one objective function to be optimized simultaneously [50]. In the signal processing context, the different objectives to be optimized are expressed as a components of a vector [48]. Here, we make use of vector $\mathbf{r}$, which contains each of the user rates to be optimized.

Since problem (2) is a MOP, there is no longer a unique optimal solution but a set of them which form the Pareto set. Mathematically, a point $x$ of the feasible set of (2) is in the Pareto set if there is no other $x^{\prime}$ such that $\mathbf{r}\left(x^{\prime}\right) \leq \mathbf{r}(x)$. The following section devotes to solve problem (2); this is, to describe the rate Pareto set.

\section{Rate pareto region of TAS licensed networks}

We can obtain an equivalent problem by manipulating the objective functions. With this, we can replace the vector objective function by $\mathbf{g}=\left[\begin{array}{lll}g_{1} & \ldots & g_{\mathrm{K}}\end{array}\right]^{\mathrm{T}}$, where

$g_{k}=\frac{\mathbf{a}_{k}^{\mathrm{T}} \mathbf{x}+\sigma^{2}}{\mathbf{a} * \mathrm{~T}_{k} \mathbf{x}+\sigma^{2}} \quad k=1, \ldots, \mathrm{K}$

Vector $\mathbf{a}_{k}$ is the $k$-th column of matrix $\mathbf{A}$ and $\mathbf{a}_{k}^{*}$ is the same vector, but in the $k$-th entry there is a 0 instead of $a_{k k}$. Clearly, (3) is a linear fractional function. Thus, (2) is a Multi-Objective Linear Fractional Problem (MOLFP) [51]. Relaying on Theorem 6.4.1 of the previous reference (see Appendix for further details), it can be seen that the 
efficient solution of (2) is included in the solutions of

$$
\begin{gathered}
\max _{\mathbf{x}} \quad \mathbf{h} \\
\text { s.t. } \quad \mathbf{A x} \leq \boldsymbol{\rho} \\
\mathbf{0} \leq \mathbf{x} \leq \mathbf{p},
\end{gathered}
$$

where

$[\mathbf{h}]_{k}=x_{k}$.

Then, the problem becomes

$$
\begin{gathered}
\max _{\mathbf{x}} \quad \mathbf{x} \\
\text { s.t. } \quad \mathbf{A x} \leq \boldsymbol{\rho} \\
\mathbf{0} \leq \mathbf{x} \leq \mathbf{p} .
\end{gathered}
$$

The solution to (6) are the power tuples on the boundary of the power feasible set. As problem (6) is equivalent to problem (2), the Pareto rates are obtained when the transmitters work at the edge of the feasible power set.

Since each component of the vector objective function is linear and the constraints are linear, problem (6) can be casted as a MOLFP [51]. These optimization problems can be solved via the multiobjective simplex method [51], which is able to find the set of efficient solutions. This method relies on the weighted-sum method scalarization technique, which transforms the MOLP into the following Linear Programming (LP) problems:

$$
\begin{gathered}
\max _{\mathbf{x}} \quad \mathbf{w}^{\mathrm{T}} \mathbf{x} \\
\text { s.t. } \quad \mathbf{A x} \leq \boldsymbol{\rho} \\
\mathbf{0} \leq \mathbf{x} \leq \mathbf{p},
\end{gathered}
$$

where for each $\mathbf{w} \in[0,1]_{1}^{K}$ so that $\sum_{i=1}^{K}[\mathbf{w}]_{i}=1$, a Pareto point of (7) is obtained and, consequently, of (2). Due to the form of the objective function, it can be observed that the Pareto set of (6) is the edge of the feasible set. As a result, the achievable rates, or the Pareto rates, are obtained when the transmitters work at the edge of the feasible power set. It is worth mentioning that his result generalizes the work in [52] where Theorem 1 presents the achievable rates of the interference channel when treating interference as noise. Our work includes the result of [52, Theorem 1], whenever the receive power constraints are not active.

Fig. 3 illustrates the TAS power feasible set and the corresponding Pareto rate region for a two user interference network. Note that the received power is the limiting constraint and not the transmit power one (e.g., in other words, this latter constraint is not active). Among all possible power-tuples on the power region boundary we are interested in the most upper right corner, $x^{*}$ in Fig. 3(a), where all communication links are active and $\mathbf{A x}=\boldsymbol{\rho}$. More precisely, this paper presents a power control that is used autonomously by each communication link in order to attain $x^{*}$. This power control was originally presented in [43]. However, neither its optimality, nor its detailed convergence, were studied. Concerning optimality, accordingly with the explanation in this section, the most upper right corner of the power region boundary attains a Pareto rate. The convergence study is done in next section, together with a study on the per-link QoS guarantee.

\section{Decentralized power control for TAS (DPC-TAS)}

We work under the premise that each receiver can only communicate feedback to its corresponding transmitter and propose a decentralized power control, which consists in the following power updating rule:

$x_{k}(n)=x_{k}(n-1)+\frac{\beta}{a_{k k}}\left(p_{\text {reg }}-m_{k}(n-1)\right) \quad k=1, \ldots, \mathrm{K}$

where $m_{k}(i)$ is the total amount of received power at the $i$-th time instant,

$m_{k}(i)=\sum_{j=1}^{\mathrm{K}} a_{j k} x_{j}(i)+\sigma^{2}$

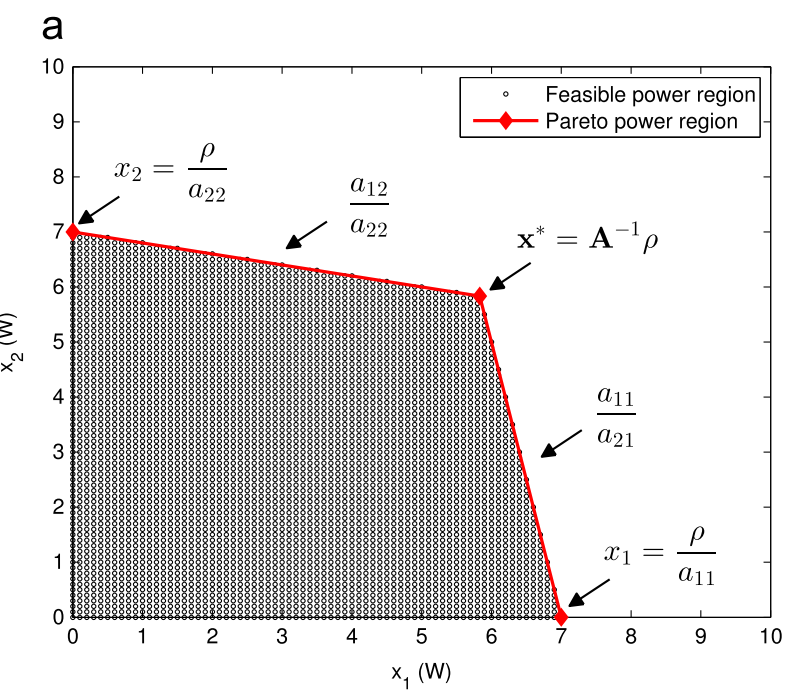

Fig. 3. Interference network with two users where $\mathbf{A}=\left[\begin{array}{ll}1 & 0.2 \\ 0.2 & 1\end{array}\right], \boldsymbol{\rho}=\left[\begin{array}{l}7 \\ 7\end{array}\right]$, Pareto rate region. b

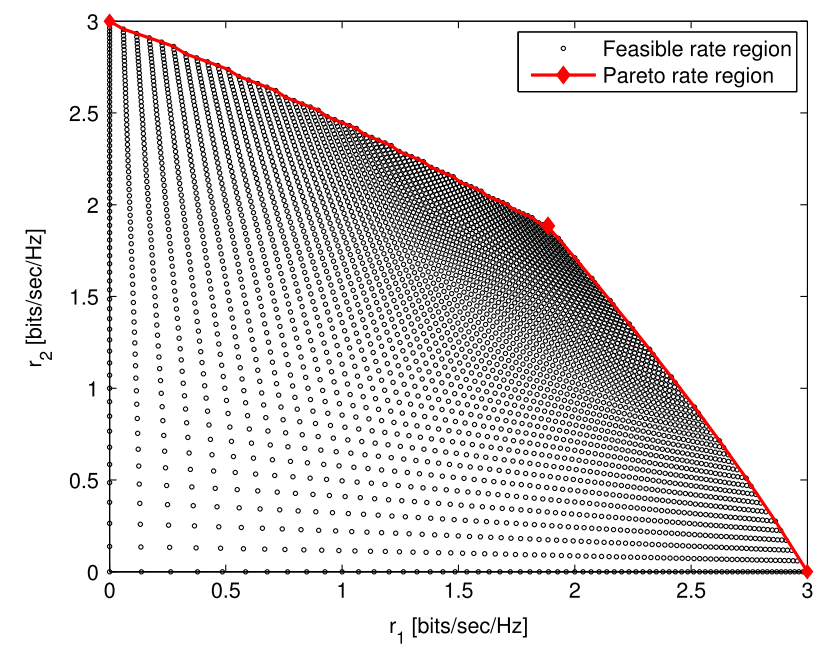


and $\beta$ is a parameter whose purpose is to control the fluctuations and convergence of the method (i.e. the socalled control gain). The receiver $k$ has to feedback the difference $\left(p_{\text {reg }}-m_{k}\right)$ to its corresponding transmitter. In this way the transmitter can adapt the transmit power following (8).

In order to justify ( 8 ) and prove that it converges to the working point $\mathbf{A x}=\boldsymbol{\rho}$, which has been justified in Section 3 as the one to be attained, note that we focus on the differential dynamic

$\frac{\mathrm{d} m_{k}}{\mathrm{~d} t}=\beta\left(p_{\text {reg }}-m_{k}\right) \quad k=1, \ldots, \mathrm{K}$

where the total received power at terminal $k, m_{k}$, is driven towards the desired mask level $p_{\text {reg }}$. In order to implement this equation only with local measurements, we assume that the $k$-th transmitter strives to evolve $m_{k}$ as if the interference contribution to the received power was not going to change. The equation for this dynamic is

$\frac{\mathrm{d} x_{k}(t)}{\mathrm{d} t}=\frac{\beta}{a_{k k}}\left(p_{\text {reg }}-m_{k}(t)\right) \quad k=1, \ldots, \mathrm{K}$

which leads to the gradient-based power control in (8), but in continuous time.

\subsection{Convergence study}

The proposed power control is a first order system, whose convergence can be studied either in the continuous or in the discrete time. We opt for the discrete one.

If we take the discrete time control in (8) and group the $K$ equations in matrix form, we obtain

$\mathbf{x}(n)=\mathbf{x}(n-1)+\beta \mathbf{D}\left(\mathbf{p}_{\text {reg }}-\mathbf{m}(n-1)\right)$

where $\mathbf{D}$ is a diagonal matrix with $[\mathbf{D}]_{i i}=a_{i i}^{-1}, \mathbf{p}_{\text {reg }}=\boldsymbol{\rho}+\sigma^{2} \mathbf{1}$ where $\mathbf{1}$ is a column vector with all its elements equal to one and

$\mathbf{m}(n-1) \triangleq \mathbf{A} \mathbf{x}(n-1)+\sigma^{2} \mathbf{1}$

By operating (12) we come up with the following expression:

$\mathbf{x}(n)=(\mathbf{I}-\beta \mathbf{D A}) \mathbf{x}(n-1)+\beta \mathbf{D} \rho$

This is a system of difference equations of first order [53] and its solution can be expressed as

$\mathbf{x}(n)=(\mathbf{I}-\beta \mathbf{D A})^{n}\left(\mathbf{x}_{0}-\mathbf{x}^{*}\right)+\mathbf{X}^{*}$

where $\mathbf{x}_{0}$ is the initial value and $\mathbf{x}^{*}$ is the steady state solution, which is obtain when $\Delta \mathbf{x}(n)=\mathbf{0}$,

$\mathbf{x}^{*}=\mathbf{A}^{-1} \boldsymbol{\rho}$

The power control converges to $\mathbf{x}^{*}$ whenever the transitory disappears. In other words, when

$\left|1-\beta \ell_{i}\right|<1 \quad \forall i$

where $\ell_{\mathrm{i}}$ are the eigenvalues of (DA).

Remark 1. Note that if (17) is satisfied for the maximum eigenvalue, it is satisfied $\forall i$. Thus, the power control converges if the following equation is satisfied:

$\beta<\frac{2}{\ell_{\max }}$
In practice, however, $\ell_{\max }$ is not known by the different communication pairs. As a consequence and in order to obtain a safe design, we propose to design $\beta$ with a small enough value in order to enforce (18). Additionally, the simulations section illustrates that, due to the normalization by $\mathbf{D}$, the maximum eigenvalue of $\mathbf{D A}$ is not very sensitive to the number of users.

As in any control, there is a set-up time, where the communication link establishes the best value for $\beta_{k}$ before data transmission. As the proposed gradient-based iteration is a first order power control, if the established value for $\beta_{k}$ produces an overshoot power in the transient time, the link will have to reduce its value. Each time a new link is established or an existing link is dropped, each existing link will experiment a transient time, which may indicate that a new value for the corresponding $\beta_{k}$ is needed. The simulation section illustrates the design of $\beta_{k}$.

We remark that the steady state solution in (16) is the solution of the power optimization in (7), whenever

$0 \leq\left[\mathbf{x}^{*}\right]_{k} \leq[\mathbf{p}]_{k}$

For this reason, the control rule in (8) has to be modified to clip the resulting power so that, for each communication, it stays below the available power $\mathbf{p}_{k}$ for each iteration $n$ :

$x_{k}^{\text {final }}(n)=\min \left(x_{k}(n), p_{k}\right) \quad \forall k$.

As in [54] it can be shown that DPC-TAS converges to

$\mathcal{T}\left(\mathbf{A}^{-1} \boldsymbol{\rho}\right)$,

where

$\mathcal{T}\left(\mathbf{A}^{-1} \boldsymbol{\rho}\right)=\min \left(p_{\text {reg }} \mathbf{1}, \mathbf{A}^{-1} \boldsymbol{\rho}\right)$,

where $\min (\cdot, \cdot)$ is considered component-wise. With [54, Proposition 2], DPC-TAS convergence is ensured. Also, in order for (16) to have a positive solution the link gains, $a_{i j}$, must keep a certain relationship as next Eq. (23) states (e.g. note in Fig. 3(a) that the value of the cross-link gains $\left(a_{i j}, i \neq j\right)$ versus the direct ones $\left(a_{i i}\right)$ play an important role in the existence of a valid power-tuple that fulfills the received power constraint with equality, $\mathbf{x}=\mathbf{A}^{-1} \boldsymbol{\rho}>\mathbf{0}$. More precisely, in [55] it is derived that, considering a positive matrix $\mathbf{A} \in \mathbb{R}^{K \times K}$ and a vector $\rho \in \mathbb{R}^{K \times 1}$, so that $\rho_{i} \gg 0, i=1, \ldots, \mathrm{K}$, if

$\forall j \rho_{j}>\sum_{\substack{i=1 \\ i \neq j}}^{\mathrm{K}} \rho_{i} \frac{a_{i j}}{a_{i i}} \underbrace{\rightarrow}_{\rho_{j}=\rho \forall j} 1>\sum_{\substack{i=1 \\ i \neq j}}^{\mathrm{K}} \frac{a_{i j}}{a_{i i}}$

then $\mathbf{A}$ is invertible and $\mathbf{A}^{-1} \boldsymbol{\rho}>\mathbf{0}$.

Remark 2. Whenever (23) is fulfilled, the weights $\mathbf{w}$ in (7) are set to one and the individual maximum power constraints are not active, then we can observe from (7) that the power control of (20) gives the maximum network power solution that allows to fulfill the regulation mask with equality and achieves a rate that is Pareto-efficient (Fig. 4).

If (23) is not fulfilled, the use of transmitters and/or receivers with multiple antennas is beneficial to design beamformers $[56-58,14,15]$ that properly attenuate the cross-link gains, $a_{i j}, j \neq i$; thus, enforcing (23). However, beamforming design is out of the scope of this paper and 
a

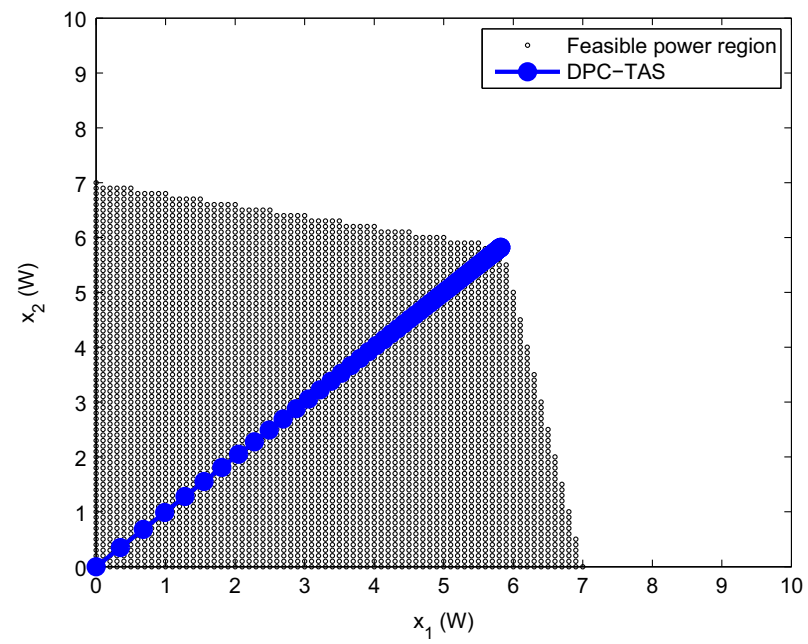

b

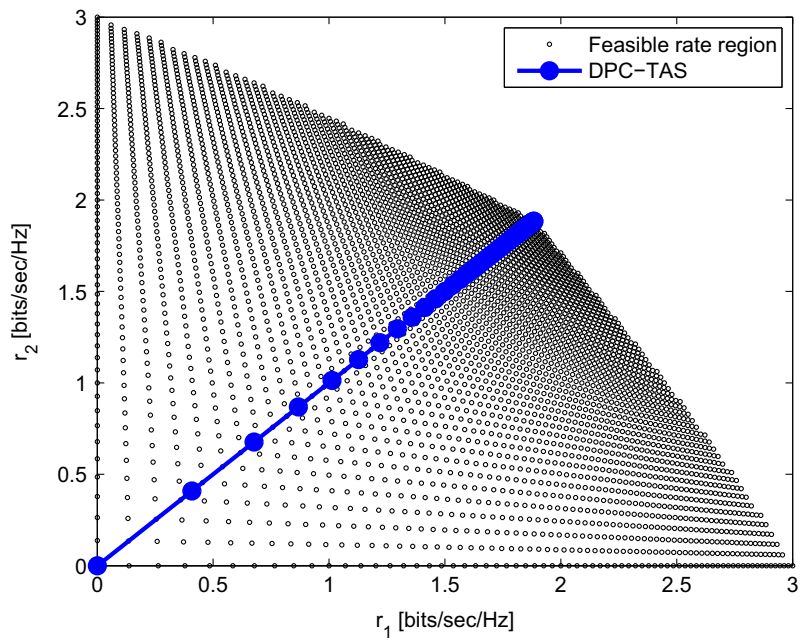

Fig. 4. Convergence of the proposed DPC-TAS for the example in Fig. 3 for $\beta_{k}=0.05 \forall k$. (a) Power feasible set and convergence of the DPC-TAS, and (b) rate feasible set and convergence of the DPC-TAS.

we leave it for future work. In this paper we deal with decentralized transmit power design, where there is no channel state information of the network available at transmission. Therefore, we can only clip the power to zero whenever the power results negative in (8). The resulting DPC-TAS power control at each iteration $n$ is

$x_{k}^{\text {final }}(n)=\max \left(0, \min \left(x_{k}(n), p_{k}\right)\right) \quad \forall k$.

As in the previous case, it can be shown that whenever the updating rule (24) is applied, the power allocation convergence is ensured to

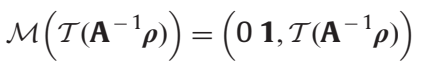

where $\max (\cdot, \cdot)$ is considered component-wise. Throughout the paper, (24) will be referred to as DPC-TAS (Decentralized Power Control for TAS). Algorithm 1 summarizes the proposed DCP-TAS approach.

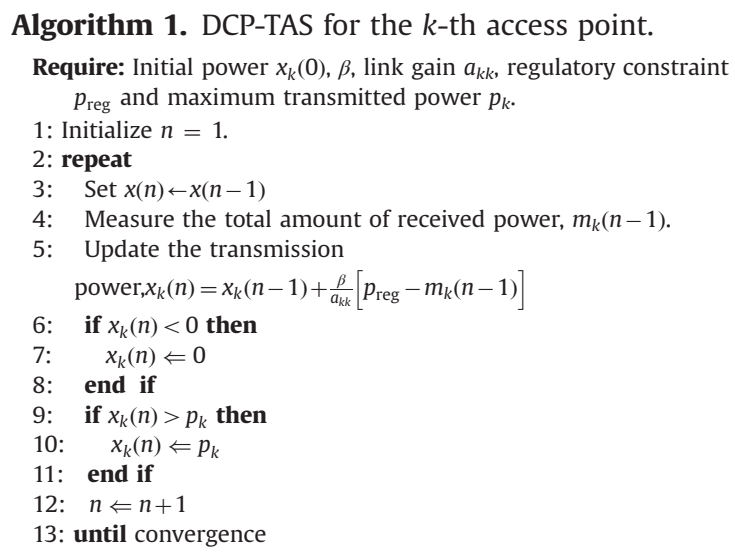

Finally, we comment that in practice the proposed power control, as any physical layer alternative, is complemented by a scheduling and access protocol. Their task, among others, is to avoid pathological situations, such as for instance having to access points that are transmitting very close-by or one user that is too far away (i.e. out of the coverage area), which could be better associated to another access point. This will help to have $a_{i i}>a_{i j}$, thus supporting condition (23). The work in [22] is a good starting point to further work this aspect as it includes network-layer solutions (e.g. combining scheduling and power control).

So far, the objective is to fulfill the regulation mask without QoS guarantees. Next section compares the rates achieved by DPC-TAS with those in a system whose goal is rate balancing or SINR-QoS.

\subsection{Minimum QoS guarantee}

Let us assume that each link has a minimum SINR threshold, $\gamma_{k}$, which must be met in order to fulfill its QoS requirements

$\frac{a_{k k} x_{k}}{\sum_{\substack{j=1 \\ j \neq k}}^{K} a_{j k} x_{j}+\sigma^{2}} \geq \gamma_{k}$

This set of equations can be set up in matrix form as

(I-F) $\mathbf{x} \geqslant \mathbf{u}$

with

$\mathbf{u}=\left[\begin{array}{llll}\frac{\sigma^{2} \gamma_{1}}{a_{11}} & \frac{\sigma^{2} \gamma_{2}}{a_{22}} & \ldots & \frac{\sigma^{2} \gamma_{\mathrm{K}}}{a_{\mathrm{KK}}}\end{array}\right]^{T}$

$[\mathbf{F}]_{j i}=\frac{\gamma_{i} a_{j i}}{a_{i i}} \delta_{j i}, \quad \forall i, j$

where $\delta_{j i}$ is the indicator function that equals 1 if $j \neq i$ and 0 otherwise. Fig. 5(a) depicts the constraint set that is defined by (27) when the network parameters are the same as those in Fig. 3.

Given a regulation mask, $\boldsymbol{\rho}=\mathbf{A x}$, the SINR thresholds contained in $\mathbf{u}$, must fulfill

(I-F) $\mathbf{A}^{-1} \boldsymbol{\rho} \geqslant \mathbf{u}$ 
a

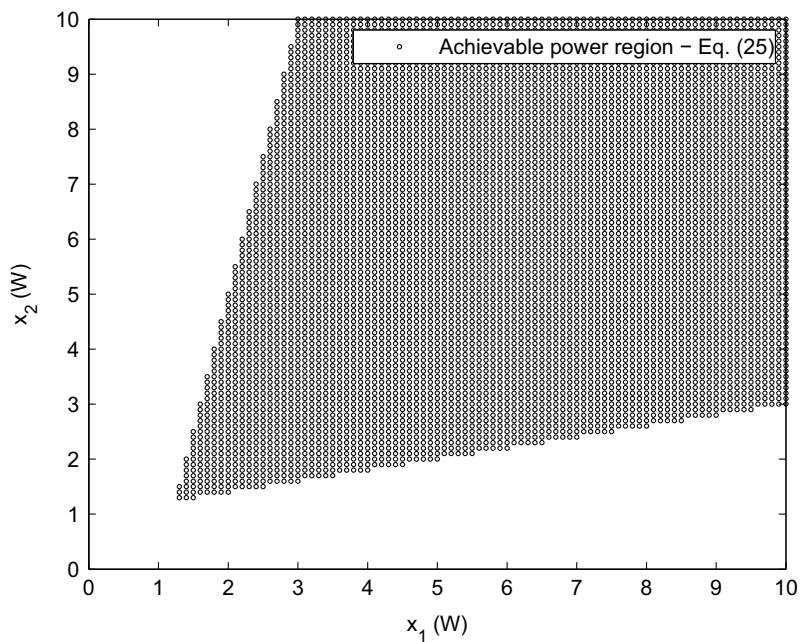

b

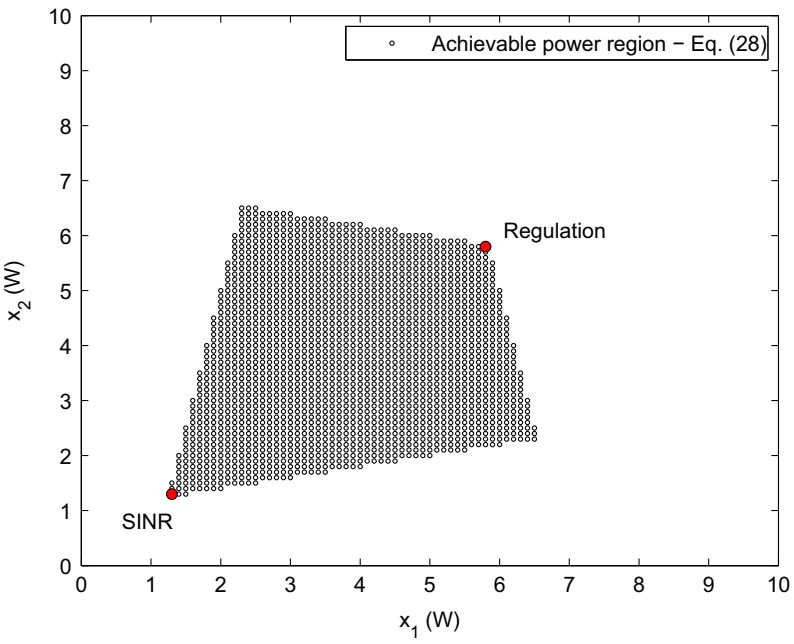

Fig. 5. Minimum QoS guarantee and DPC-TAS for the example in Fig. 3 for $\gamma=\left[\begin{array}{l}1 \\ 1\end{array}\right]$. (a) SINR constraint set, and (b) Intersection of regulation and SINR sets. Red points indicate the two vertices that meet with equality either the regulation or the SINR constraints. (For interpretation of the references to color in this figure caption, the reader is referred to the web version of this paper.)

Foschini and Miljanic proposed in [18] a decentralized power control in order to simultaneously satisfy all SINR thresholds in the network. As long as the maximum modulus eigenvalue of $\mathbf{F}$ is real, positive and simple, and $(\mathbf{I}-\mathbf{F})^{-1}$ exists, there is a first order power control that converges to attain the required SINR thresholds with minimum network power, which can be implemented autonomously by each link. As it happens with the regulation constraints in Section 4.1, convergence to the QoS requirements of [18] depends also on the link gains.

In contrast to [18], note that the goal of the proposed decentralized algorithm DPC-TAS in (24) is to implement a power control that is Pareto-efficient in network-rate terms without considering QoS. In order to incorporate SINR-QoS constraints we should incorporate (27) into the LP that is formulated in (7). This is

$$
\begin{array}{rl}
\max _{\mathbf{x}} & \mathbf{x} \\
\text { s.t. } \quad \mathbf{A} \mathbf{x} \leq \boldsymbol{\rho} \\
(\mathbf{I}-\mathbf{F}) \mathbf{x} \geqslant \mathbf{u}, \\
\mathbf{0} \leq \mathbf{x} \leq \mathbf{p} .
\end{array}
$$

This latter optimization problem provides the achievable rates of a TAS licensed system under SINR restrictions. Evidently, the feasible set of (30) differs from that in (6). This can be observed in Fig. 5. As a matter of fact, the rate Pareto set obtained from (30) ensures that all users will have the required SINR s. Furthermore, note that whenever the feasible set is convex (i.e. the minimum SINR constraints contain the MURC), the proposed power control Algorithm 8 leads to a power tuple that lies on the MURC, which is rate optimal and in addition fulfills the SINR requirements. In other words, DPC-TAS mechanism ensures the SINR requirements without any additional modification.
Note that there are two possible situations that a communication pair can face. One situation is to receive total power above the regulation mask. In this case, this communication pair will lower its transmitting power as (8) indicates. The other situation is to be below the regulation mask. Then an additional power control, as the one in [18], can check if the QoS for that communication pair is fulfilled. If it is not the case, then it can aim for it. However, as it is a decentralized control and one communication link does not have knowledge about the channel state information of the other links, with this increase/decrease in power we cannot guarantee that all communication links meet the QoS constraints within the regulation limits. In other words, there is no way to guarantee the convergence to the vertix of the constraint set that offers the optimal solution for (30). The convergence of a power that is non-linearly controlled by two different equations (i.e. the one in (24) to guarantee the regulation mask and the one in [18] to guarantee QoS, as we are suggesting in this explanation) is complex and nontrivial. Power control in a communication network should be practical; thus, clean and simple. Otherwise, it is advisable to resort to additional degrees of freedom, as for instance, the spatial selectivity or beamforming that has been commented before, in order to attain the required QoS.

This section has studied in detail the power control that was originally presented in [43]. More specifically, it studies DPC-TAS convergence and frames the algorithm together with other power control that attains QoS. The conclusion is that DPC-TAS is a simple control that attains the regulation mask and also, under certain channel gains, can attain per-link QoS. In order to get more insight into the good features of DPC-TAS, next section introduces a pricing strategy for decentralized power control in a TAS regulated system. We show that the linear dynamic pricing, despite being intuitively easy to understand and implement, it is less practical for the interference channel 
than the DPC-TAS that is proposed in Section 4. The design of a suitable non-linear pricing is open for future work. Note that one of the interesting aspects of pricing mechanisms is that they give a new and enlarged framework to deal with possible situations of users misbehavior. These situations are, however, out of the scope of the present paper.

\section{Pricing for TAS}

This paper focuses on strategies where the power is allocated autonomously by each link itself and each link/ user cares only about maximizing its own utility. In microeconomic models, when users want more rather than less of a good they are doing rational choices in a market context. Game theory emphasizes on the mathematical modeling among rational agents or players, who are selfish by nature. Pricing can be introduced to control this selfish behavior. Our interest is to design a simple pricing power algorithm and compare it with DPC-TAS. For this reason control theory tools are used for its analysis instead of game-theoretic or convex optimization, which are the usual tools whenever pricing is introduced. More specifically, we are interested in the power control problem with linearly coupled constraints. In [46] the reader can find several game-theoretic tools to analyze a broad family of games played by resource-constrained players. In [44] a pricing algorithm is presented to cope with linear interference power constraint in cognitive networks. Very interesting is also [59], where the authors study QoSaware distributed resource (i.e. power and spectrum) sharing using a game-theoretic approach.

In our work, where rate optimization is the ultimate goal, we depart from the following basic problem:

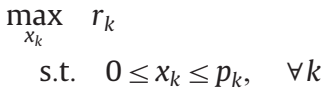

Note that users do not cooperate and, in spite of creating more interference, each user would like to maximize their individual rate by transmitting at full power, $\mathbf{x}^{*}=\mathbf{p}$. This solution is identified as a Nash Equilibrium and can be seen as a pure selfish user response. However, for the TAS power control we have to incorporate the regulation constraint. In order to obtain a simple first order control, we propose to penalize the rate utility of each user $k$ with the following pricing mechanism that is linear on the transmit powers as (32) indicates

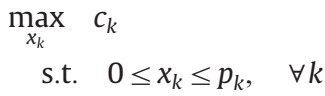

where $c_{k}=r_{k}-\pi_{k}^{-1} a_{k k} x_{k}$ and $\pi_{k}$ are the prices, which mathematically can be understood as additional degrees of freedom that are designed to meet the desired operating point. If the aim is to meet the regulation mask, then we should

$$
\begin{aligned}
\text { find } & \pi_{k} \\
\text { s.t. } & m_{k}=p_{\text {reg. }} . \quad \forall k
\end{aligned}
$$

When looking only at the $\operatorname{cost} c_{k}$, note that if the prices $\pi_{k}=\infty$, transmitting with full power is the optimal response of each user; thus, $\pi_{k}<\infty$ motivates to avoid fullpower transmission. The strategy of using $\pi_{k}^{-1}$ instead of $\pi_{k}$ is justified in what follows.

For each user, the optimal power control must maximize its rate without violating the regulation mask. Therefore, the optimal solution is obtained by deriving (32) and equating to zero

$\frac{\partial c_{k}}{\partial x_{k}}=0 \rightarrow x_{k}^{*}=\frac{1}{a_{k k}}\left[\pi_{k}-\left(\sigma^{2}+\sum_{\substack{j=1 \\ j \neq k}}^{\mathrm{K}} a_{j k} x_{j}\right)\right] \quad \forall k$,

which depends linearly on the prices. Note that in (34) the factor $\frac{1}{\ln 2}$ which comes from the derivative of $\log _{2}$ has been incorporated to the prices. This equation can be used to obtain the desired powers in an iterative way as follows:

$$
\begin{aligned}
x_{k}(n) & =\frac{1}{a_{k k}}\left[\pi_{k}-\left(\sigma^{2}+\sum_{\substack{j=1 \\
j \neq k}}^{\mathrm{K}} a_{j k} x_{j}(n-1)\right)\right] \\
& =\frac{1}{a_{k k}}\left[\pi_{k}-\left(m_{k}(n-1)-a_{k k} x_{k}(n-1)\right)\right] \\
& =x_{k}(n-1)+\frac{1}{a_{k k}}\left(\pi_{k}-m_{k}(n-1)\right) \quad \forall k,
\end{aligned}
$$

which can be written in matrix notation as follows:

$$
\begin{aligned}
\mathbf{x}(n) & =\mathbf{x}(n-1)+\mathbf{D}\left(\pi-\left(\mathbf{A x}(n-1)+\sigma^{2} \mathbf{1}\right)\right) \\
& =\mathbf{x}(n-1)+\mathbf{D}(\pi-\mathbf{m}(n-1)) \\
& =(\mathbf{I}-\mathbf{D A}) \mathbf{x}(n-1)+\mathbf{D}\left(\pi-\sigma^{2} \mathbf{1}\right)
\end{aligned}
$$

This set of power controls is a system of difference equations of first order, whose steady state solution $\mathbf{x}^{*}$ is obtained for $\Delta \mathbf{x}(n)=\mathbf{0}$,

$\Delta \mathbf{x}(n)=\mathbf{0} \rightarrow \boldsymbol{\pi}=\mathbf{A} \mathbf{x}^{*}+\sigma^{2} \mathbf{1}$

where $\pi=\left[\begin{array}{llll}\pi_{1} & \pi_{2} & \ldots & \pi_{\mathrm{K}}\end{array}\right]^{\mathrm{T}}$. In order to ensure the positive power control, an analogous condition to that in (23) should be fulfilled. From the game theory point of view, this set of power controls can be seen as a set of noncooperative games, which always admits at least one NE power allocation and one possible NE is (37). This NE will be unique and instead of resorting to classical control theory to study its convergence, non-cooperative game theory could be applied and, with it, conclude that (36) is guaranteed to converge to the unique NE whenever (34) is a standard function. That means that (34) is: positive, monotone and scalable (see [40]). However, in the equilibrium point of (37) the value of the prices is still to be set; thus, requiring further study to solve the final convergence. In what follows classical control is the mainstream for this study.

The objective of the pricing, $\pi_{j} \forall j$, in the proposed game is to enforce the NE power control to the desired point in (33). As the goal is to obtain a decentralized power allocation, we consider the classical subgradient with a constant stepsize $\mu$

$\boldsymbol{\pi}(n)=\boldsymbol{\pi}(n-1)+\mu\left[\mathbf{m}(n-1)-\mathbf{p}_{\mathrm{reg}}\right]$

The obtained power control is summarized in Algorithm 2 and is named Pricing-TAS. We remark that no pricing announcement to the network is needed. 
Algorithm 2. Pricing-TAS for the $k$-th access point.

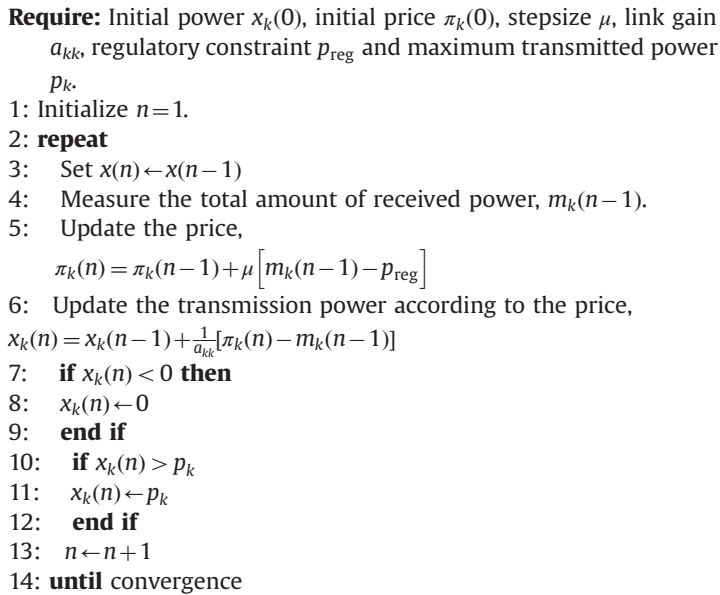

To study the convergence we again identify a system of difference equations of first order: We substitute $\pi(n)$ into (36); thus, obtaining

$$
\begin{aligned}
\mathbf{x}(n) & =\mathbf{x}(n-1)+\mathbf{D}[\pi(n)-\mathbf{m}(n-1)] \\
& =\mathbf{x}(n-1)+\mathbf{D}\left[\pi(n-1)+(\mu-1) \mathbf{m}(n-1)-\mu \mathbf{p}_{\mathrm{reg}}\right] \\
& =\mathbf{x}(n-1)+\mathbf{D}\left[\pi(n-1)+(\mu-1) \mathbf{A} \mathbf{x}(n-1)-\mu \rho-\sigma^{2} \mathbf{1}\right] \\
& =[\mathbf{I}+(\mu-1) \mathbf{D A}] \mathbf{x}(n-1)+\mathbf{D} \pi(n-1)-\mathbf{D}\left[\mu \rho+\sigma^{2} \mathbf{1}\right]
\end{aligned}
$$

We group (38) and (39) as

$\nu(n)=\mathbf{B} \nu(n-1)+\mathbf{b}$

with

$\nu(n)=\left[\begin{array}{ll}\mathbf{x}(n) & \pi(n)\end{array}\right]^{\mathrm{T}}$

$\mathbf{B}=\left[\begin{array}{ll}\mathbf{I}+(\mu-1) \mathbf{D A} & \mathbf{D} \\ -\mu \mathbf{A} & \mathbf{I}\end{array}\right]$

$\mathbf{b}=-\left[\begin{array}{ll}\mathbf{D}\left[\mu \boldsymbol{\rho}+\sigma^{2} \mathbf{1}\right] & \mu \boldsymbol{\rho}\end{array}\right]^{\mathrm{T}}$

Following the same reasoning as in Section 4.1 (i.e. (18)), we can say that this new power control converges whenever the maximum eigenvalue of matrix $\mathbf{B}$ has modulo less than one. The steady state solution is obtained for $\Delta \boldsymbol{\nu}(n)=\mathbf{0}$, which means $\Delta \mathbf{x}(n)=\mathbf{0}$ in (39) and $\Delta \boldsymbol{\pi}(n)=\mathbf{0}$ in (38). As a consequence

$\Delta \mathbf{x}(n)=\mathbf{0} \rightarrow \mathbf{D} \pi^{*}+(\mu-1) \mathbf{D} \mathbf{A} \mathbf{x}^{*}=\mathbf{D}\left[\mu \boldsymbol{\rho}+\sigma^{2} \mathbf{1}\right]$

and

$\Delta \pi(n)=\mathbf{0} \rightarrow \rho=\mathbf{A} \mathbf{x}^{*}$

Combining the two equations it results that the steady state solution (i.e. for $n \rightarrow \infty$ ) is

$\mathbf{A} \mathbf{x}^{*}=\boldsymbol{\rho}=\mathbf{p}_{\text {reg }}-\sigma^{2} \mathbf{1}$,

which coincides with the steady state solution that was obtained in (37), where $\pi$ was not computed iteratively. Pricing incorporates a certain social welfare due to the regulation mask constraint. In other words, although each user can only modify its own transmit power, the rate of each user is penalized if the received power by each user is above the regulation mask.

In contrast to the convergence of DPC-TAS in Section 4 (i.e. (17)), the convergence of the Pricing-TAS power control that is obtained with (39) and (38) is more difficult to guarantee. The reason is that in this latter control, the value of the gain $\mu$ has to be computed so that matrix $\mathbf{B}$ in (41b) presents a maximum eigenvalue with modulo less than one and this is not the straightforward design of (17). In addition, as the number of simultaneous communication links increases, the maximum eigenvalue of $\mathbf{B}$ notably changes. This requires a redesign of $\mu$ depending on the number of users. However, in a decentralized design, the number of total users is not known by each communication link. The simulation section illustrates these problems with some examples.

In summary, the basic Pricing-TAS power control that we propose converge to the same optimal solution as the DPC-TAS that is studied in Section 4. However, the problem of Pricing-TAS resides in the stability, especially when the number of users increases and we have shown it analytically. We leave for future work the design of a non-linear pricing that improves the classical power control that we propose for TAS. Note that interest of pricing and the game theoretic point of view is that they open new and powerful alternatives to design resource allocation strategies that properly penalize whenever the users misbehave. In decentralized designs users may hide their private interests or true utility functions to each other in order to overtake the other users in performance. This is an undesired situation from the network perspective and should be solved. Game theory is a useful tool and [40] and references therein are good examples of that. However, convergence may become difficult to study and analyze.

\section{Numerical results}

In order to evaluate our theoretical findings, Fig. 6 (a) and (b) show the emitted and received power evolution for the proposed DPC-TAS and the proposed Pricing-TAS power control, respectively, and for a two-user asymmetric network with

$\mathbf{A}=\left[\begin{array}{ll}1 & 0.2 \\ 0.3 & 1\end{array}\right], \quad \mathbf{p}_{\text {reg }}=\left[\begin{array}{l}7 \\ 7\end{array}\right], \quad \mathbf{p}=\left[\begin{array}{l}10 \\ 10\end{array}\right], \quad \sigma^{2}=1$.

In both cases, the initial power allocation has been set to $0 \mathrm{dBm}$. In the case of the Pricing-TAS, the initial pricing $\boldsymbol{\pi}(0)$ has been set to $\mathbf{0}$ as well. Fig. 6(a) shows the performance of DPC-TAS with $\beta=0.05$ and $\beta=0.1$, while Fig. 6 (b) illustrates the behavior of Pricing-TAS for step-size $\mu=-0.2$ and $\mu=-0.1$. In so doing, both methods converge in a similar number of iterations. From the comparison of Fig. 6(a) with Fig. 6(b), it is confirmed that the proposed DPC-TAS converges to the same optimal point than the Pricing-TAS approach.

The emitted and received power evolution with DPCTAS and with the Pricing-TAS power control are compared in Fig. 7 for a three-user network and for the same initial conditions and $\beta$ and $\mu$ values considered in the previous case. In particular, Fig. 7(a) and (b) show the transient 
a
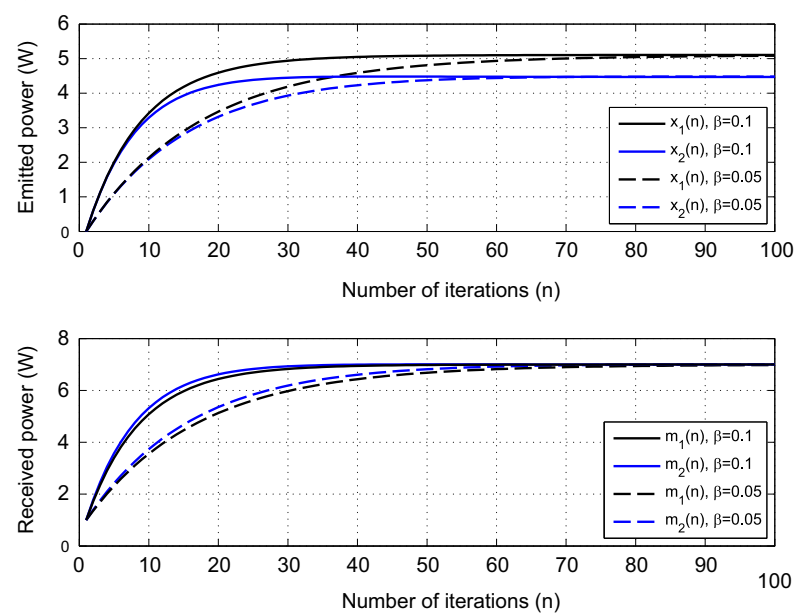

b
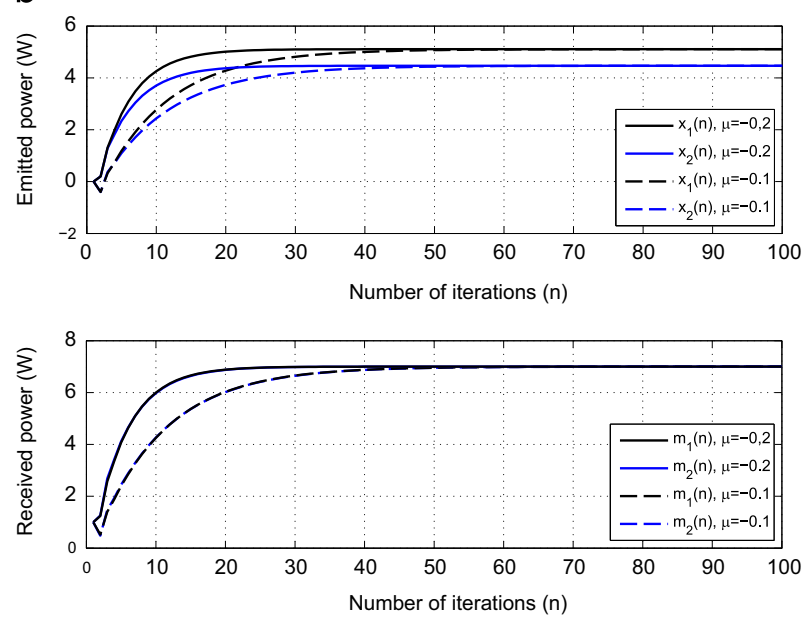

Fig. 6. Emitted and received power evolution for a 2-user asymmetric network: (a) DPC-TAS, and (b) Pricing-TAS.

a
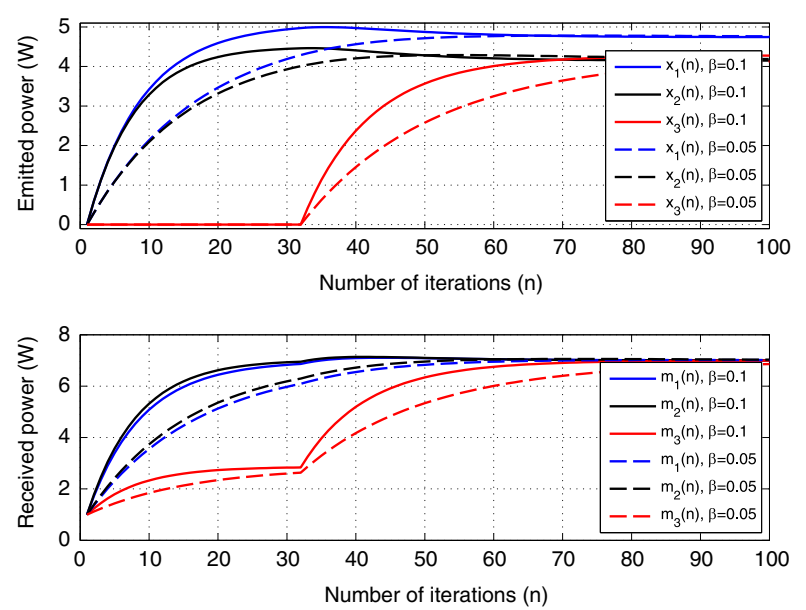

b
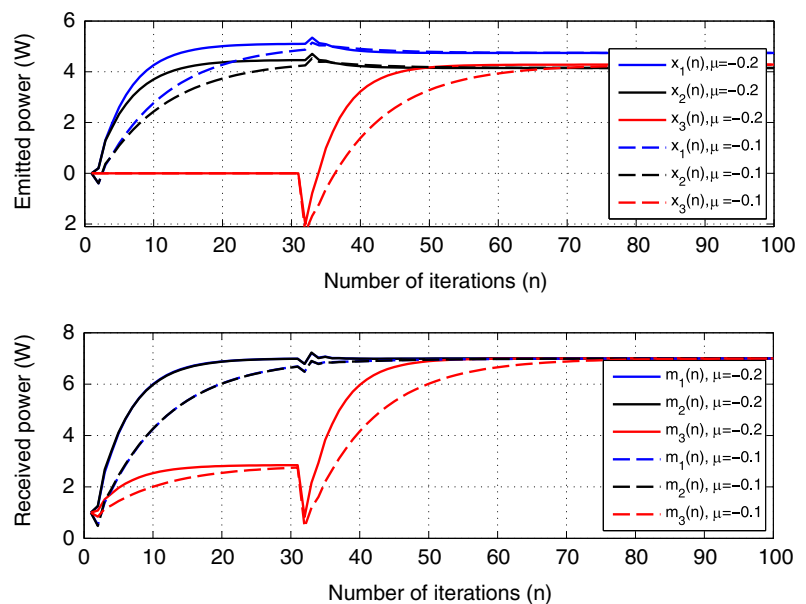

Fig. 7. Emitted and received power evolution for a 3-user asymmetric network: (a) DPC-TAS, and (b) Pricing-TAS.

effect of DPC-TAS and Pricing-TAS, respectively, when a third user joins the network at iteration $n=30$. It can be observed that the proposed DPC-TAS solution smoothly adapts to the network conditions while Pricing-TAS experiences more difficulties. In Fig. 7 we have considered the following parameters:

$$
\mathbf{A}=\left[\begin{array}{lll}
1 & 0.2 & 0.1 \\
0.3 & 1 & 0.1 \\
0.1 & 0.3 & 1
\end{array}\right], \quad \mathbf{p}_{\text {reg }}=\left[\begin{array}{l}
7 \\
7 \\
7
\end{array}\right], \quad \mathbf{p}=\left[\begin{array}{l}
10 \\
10 \\
10
\end{array}\right], \quad \sigma^{2}=1
$$

The effect of limited maximum emitted power is evaluated in Fig. 8 considering the same 3-user network as in Fig. 7, but with $\mathbf{p}=\left[\begin{array}{lll}10 & 10 & 3\end{array}\right]^{\mathrm{T}}$. As expected, in both methods the third user, whose maximum emitted power is well below the power regulation limit $\left(p_{3}<p_{\text {reg }}\right)$, converges to a lower value compared to the targeted power regulation limit.
Fig. 9(a) and (b) compares the two methods when the number of users increases up to five. For the sake of clarity, only the power evolution of 2 of the 5 users have been plotted in Fig. 9. It has been used the parameters and initial values of (46), and the extra users have been modeled as $a_{i i}=1, a_{i j}=a_{j i}=0.2, p_{\text {reg }}=7$ and $p=10$. It can be observed that the stability of the power updating rule method remains invariable. However, the announced convergence drawback for the Pricing-TAS approach is highlighted in this case.

Fig. 10(a) illustrates the stability of both methods, by evaluating the absolute value of the maximum eigenvalue of the matrix (I- $\beta$ DA) for DCP-TAS and the matrix $\mathbf{B}$, for Pricing-TAS with regard to the number of users. As in the previous examples, we used the same parameters and initial values of Fig. 7, and we modeled the extra users as $a_{i i}=1, a_{i j}=a_{j i}=0.2, p_{\text {reg }}=7$ and $p=10$. Again, we considered $\beta=0.05$ and $\beta=0.1$ for the DPC-TAS, and step-size $\mu=-0.2$ and $\mu=-0.1$ for the Pricing-TAS. As it was 
a
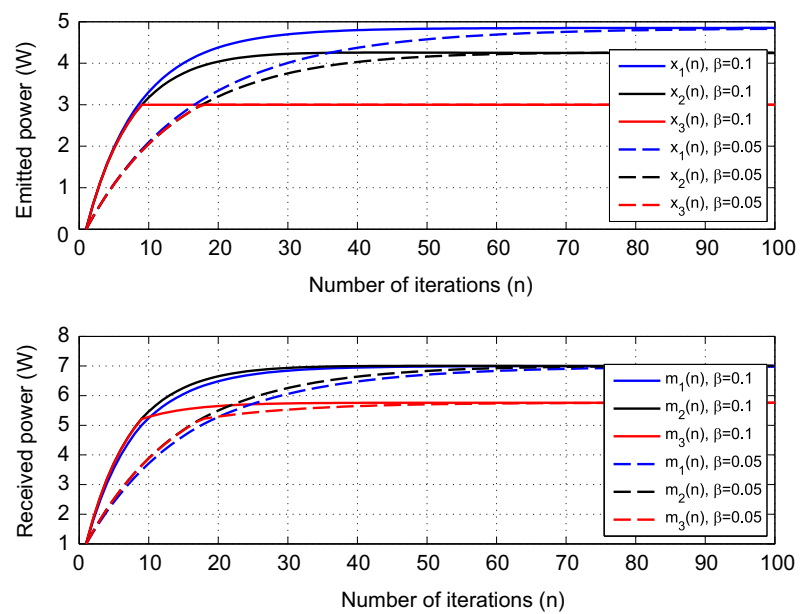

b
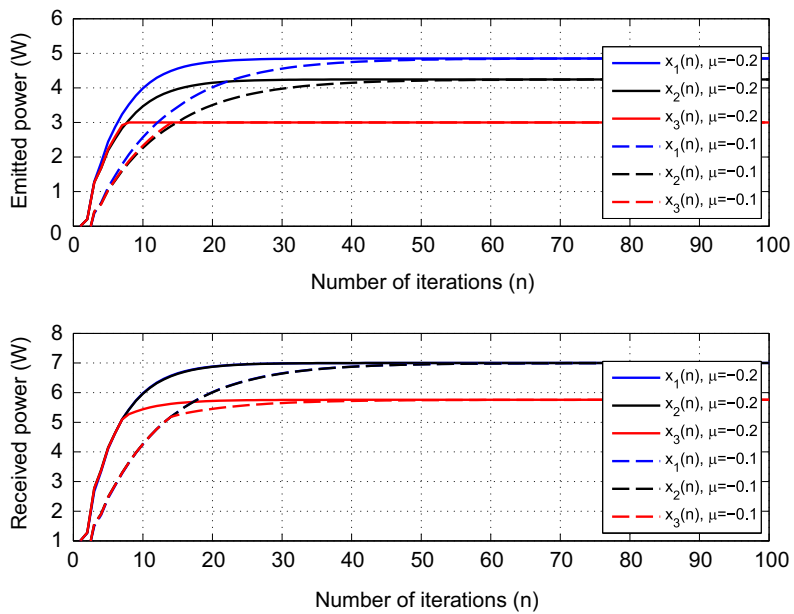

Fig. 8. Emitted and received power evolution for a 3-user asymmetric network with $\mathbf{p}=\left[\begin{array}{lll}10 & 10 & 3\end{array}\right]^{\mathrm{T}}$ : (a) DPC-TAS, and (b) Pricing-TAS.

a
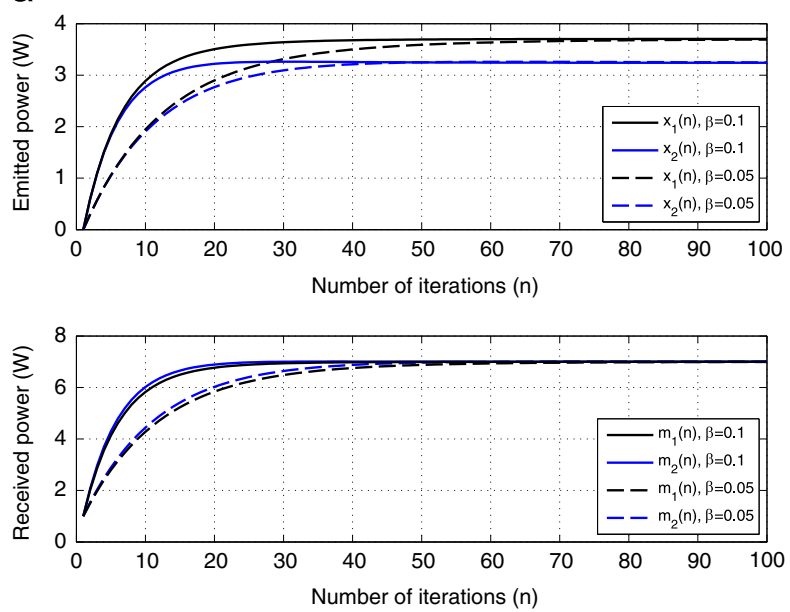

b
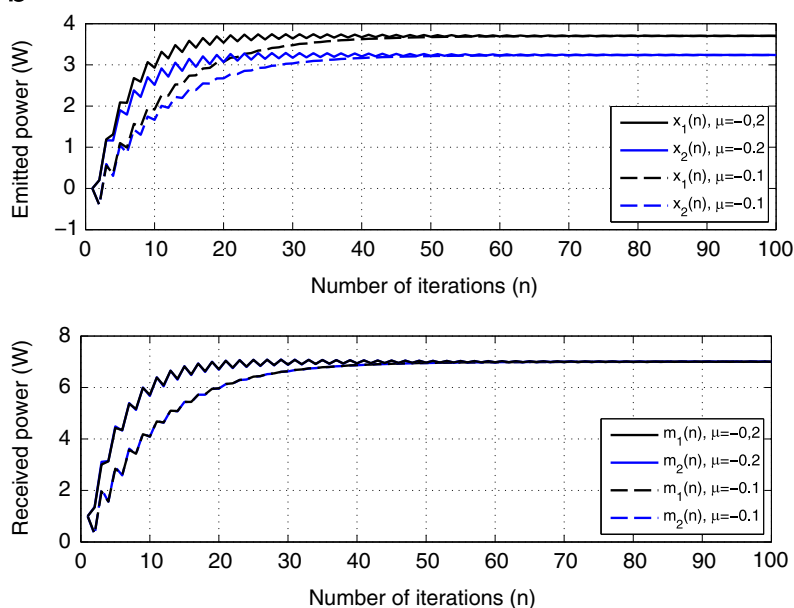

Fig. 9. Emitted and received power evolution for a 5-user asymmetric network: (a) DPC-TAS, and (b) Pricing-TAS.

explained before, although the number of channels increases, in DPC-TAS the selection of $\beta$ that fulfills the stability criterion of (18) is not critical because the modulo of the maximum eigenvalue does not depend on the network size. Nevertheless, the second method becomes unstable between 5 and 6 users for both values of $\mu$, which is clearly highlighted in Fig. 9(b). To better show this instability issue of the Pricing-TAS, Fig. 10(b) illustrates the phenomenon when a channel becomes marginally stable (stable but with a noticeable ripple) by including a new user in the network. Fig. 10(b) shows the emitted and received power evolution for a 4-user network which, at the iteration number 30 incorporates a fifth user. As it can be seen, the rippled signal overshoots the target limit after $n=30$. This situation should be detected by the hardware to proceed to reduce the gains in the correspondent channel, and recover the stability again.
Finally, although the most usual scenario is that sketched in Fig. 7, where 3 communications are colliding, Fig. 11 illustrates the effect of a bigger size network. Fig. 11 (a) shows the emitted and received power evolution with DPC-TAS for a 40-user network compared to a 5-user network. Again, parameters and initial values are the same to those of Fig. 7, and we modeled the extra users as $a_{i i}=1, a_{i j}=a_{j i}=0.2, p_{\text {reg }}=7$ and $p=10$. In Fig. 11(a) it can be observed that the transient time of the received power decreases with the number of users. Fig. 11(b) depicts the time constant in number of iterations of DPC-TAS method with regard to the number of users, for both $\beta=0.1$ and $\beta=0.05$. Clearly, higher values of $\beta$ tend to speed up the convergence time. However, one cannot choose an arbitrary high value of $\beta$ because the highest value of $\beta$ is upper-bounded by (18). 
a

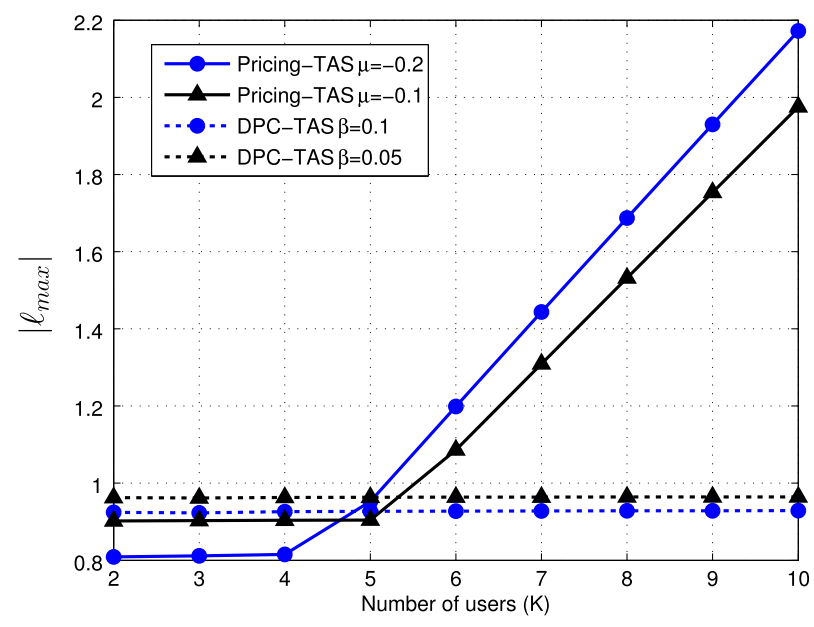

b
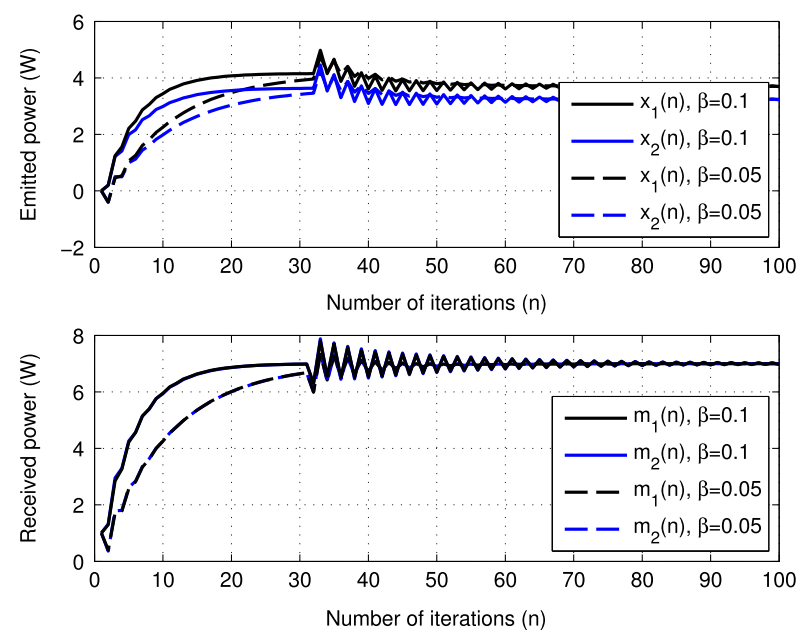

Fig. 10. Inestability of Pricing-TAS: (a) Comparison of the maximum eigenvalue of the matrix (I- $\beta$ DA $)$ for DPC-TAS and the matrix B for Pricing-TAS, and (b) Pricing-TAS emitted and received power evolution when number of users increases from 4 to 5.

a
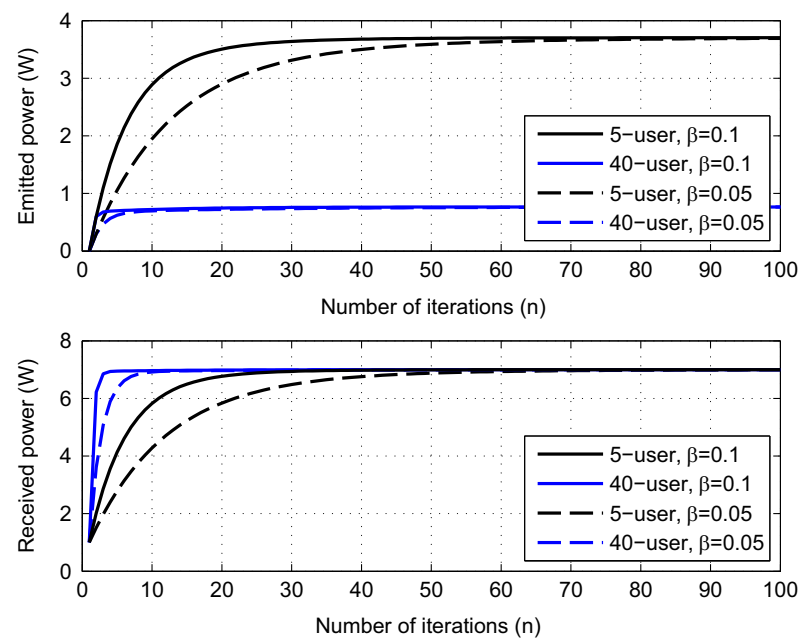

b

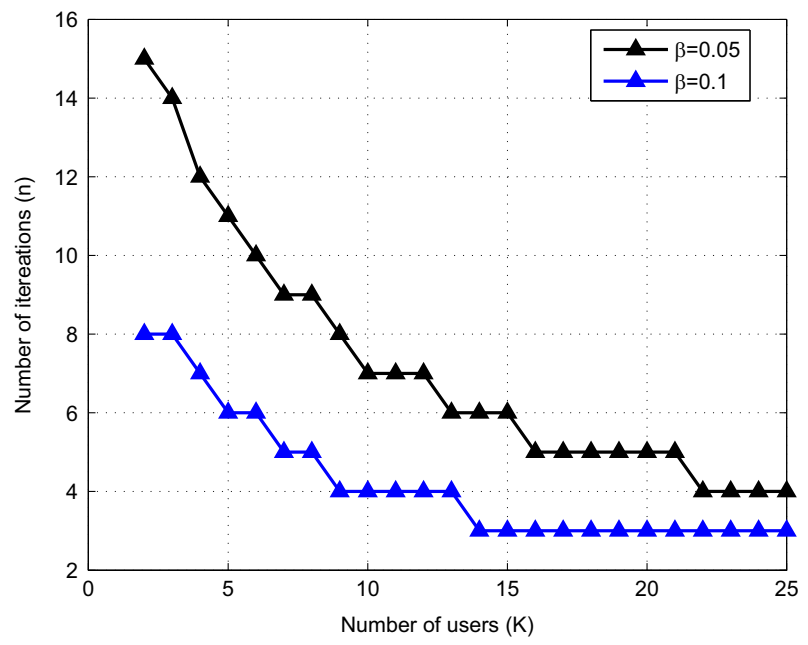

Fig. 11. Effect of network size in DPC-TAS: (a) Emitted and received power evolution of a 40-user network compared with a 5-user network, and (b) convergence time constant in number of iterations with respect to the network size.

\section{Conclusions and further work}

This paper proposes a regulation mechanism for improving the spectral efficiency of different spectrumsharing networks. Considering the TAS licensing system, with received power constraints, we provide the optimal decentralized power policy by considering the problem as a multicriteria optimization problem. We provide its convergence study and comparison with minimum QoS solutions. In addition, the paper studies a pricing algorithm for TAS; thus, framing the problem in a game theoretic framework and opening the door to enlarge the proposed power control in order to incorporate user misbehavior. The proposed pricing control helps also to compare two different focuses to solve a power allocation problem, i.e. from a basic power control perspective or from an utility optimization one. Future work on this focus, together with the design of suitable beamforming for TAS and scheduling and access mechanisms is worth to pay attention to. Numerical results show the performance of our proposal.

\section{Appendix A}

The aim of this appendix is to show the Pareto set equivalence between problem

$$
\begin{aligned}
& \max _{\mathbf{x}} \quad \mathbf{g} \\
& \text { s.t. } \quad \mathbf{A x} \leq \boldsymbol{\rho} \\
& \mathbf{0} \leq \mathbf{x} \leq \mathbf{p},
\end{aligned}
$$


and (4). Similarly to [51, Theorem 6.4.1] and relaying on [60] it is possible to obtain an equivalent multicriteria problem with the same Pareto set with the following set of objective functions:

$[\mathbf{t}]_{k}=\mathbf{a}_{k}^{\mathrm{T}} \mathbf{x}-\left(1+a_{k k} p_{k}\right) \mathbf{a}_{k}^{* \mathrm{~T}} \mathbf{x}$.

Using Dinkelbach's theorem in [60] an optimal solution of $[\mathbf{t}]_{k}$ is an optimal solution of $[\mathbf{g}]_{k}$ and viceversa. By simple inspection and since we are maximizing the vector objective function, the $k$ objective function will always yield into a solution whose entries $i \neq k$ for $i=1, \ldots N$ are zero. Under this context, each objective function can be rewritten so that

$[\mathbf{h}]_{k}=x_{k}$.

\section{References}

[1] S. Haykin, Cognitive radio: brain-empowered wireless communications, IEEE J. Sel. Areas Commun. 23 (2) (2005) 201-220.

[2] Y. Liang, H. Chen, J. Mitola, P. Mahonen, R. Kohno, J. Reed, L. Milstein, Guest editorial-cognitive radio: theory and application, IEEE J. Sel. Areas Commun. 26 (January (1)) (2008) 1-4.

[3] A. Goldsmith, S.A. Jafar, I. Maric, S. Srinivasa, Breaking spectrum gridlock with cognitive radio: an information theoretic perspective, ProcIEEE 97 (May (5)) (2009) 894-914.

[4] K. Pelechrinis, P. Krishnamurthy, M. Weiss, T. Znati, Cognitive radio networks: realistic or not? SIGCOMM Comput. Commun. Rev. 43 (April (2)) (2013) 44-51.

[5] Authorized Shared Access (ASA): an evolutionary spectrum authorization scheme for sustainable economic growth and consumer benefit, Ingenious Consulting Networks, commissioned by Qualcomm and Nokia.

[6] A. de Vany, R. Eckert, C. Meyers, D. OHara, R. Scott, A property system for market allocation of the electromagnetic spectrum: a legaleconomic-engineering study, JSTOR Stanford Law Rev. 21 (June (6) (1969) 1499-1561.

[7] A. de Vany, Implementing a market-based spectrum policy, J. Law Econ. 41 (1998) 627-646.

[8] M. Gastpar, Gaussian multiple-access channels under receivedpower constraints, in: IEEE Information Theory Workshop, San Antonio, Texas, USA, October, 2004, pp. 452-457.

[9] Y. Xing, C. Mathur, M. Haleem, R. Chandramouli, K. Subbalakshmi, Dynamic spectrum access with qos and interference temperature constraints, IEEE Trans. Mobile Comput. 6 (4) (2007) 423-433, http: //dx.doi.org/10.1109/TMC.2007.50. ISSN 1536-1233.

[10] A.G. Marqués, E. Dall'Anese, G.B. Giannakis, Cross-layer optimization and receiver localization for cognitive networks using interference tweets, IEEE J. Sel. Areas Commun. 32 (3) (2014) 641-653, http://dx doi.org/10.1109/JSAC.2014.1403009.

[11] R. Knopp, P. Humblet, Information capacity and power control in single-cell multiuser communications, in: IEEE International Conference on Communications (ICC), Seattle, Washington, USA, June 1995.

[12] E. Bjornson, E. Jorswieck, Optimal resource allocation in coordinated multi-cell systems, in: Foundations and Trends in Communications and Information Theory, Now Publishers, Boston-Delft, 2013.

[13] M. Wolkerstorfer, T. Nordstrom, Comparative simulation study of fast heuristics for power control in copper broadband networks, Signal Process. 104 (November) (2014) 437-449.

[14] H. Li, T. Liu, J. Zhang, K. Wong, Worst-case performance optimization for robust power control in downlink beamforming, Signal Process. 93 (December (12)) (2013) 3278-3289.

[15] M. Razaviyayn, M. Hong, Z. Luo, Linear transceiver design for a MIMO interfering broadcast channel achieving max-min fairness, Signal Process. 93 (12) (2013) 3327-3340.

[16] R. Yates, A framework for uplink power control in cellular radio systems, IEEE J. Sel. Areas Commun. 13 (September (7)) (1995) $1341-1347$.

[17] H. Boche, M. Schubert, A unifying approach to interference modeling for wireless networks, IEEE Trans. Signal Process. 58 (May (6)) (2010) 3282-3297.
[18] G. Foschini, Z. Miljanic, A simple distributed autonomous power control algorithm and its convergence, IEEE Trans. Veh. Technol. 42 (November (4)) (1993) 641-646.

[19] H. Iiduka, Fixed point optimization algorithm and its application to power control in CDMA data networks, Math. Programm. Ser. A 133 (June (1-2)) (2012) 227-241.

[20] C. Wei-Tan, Wireless network optimization by Perron-Frobenius theory, in: Conference in Information Sciences and Systems (CISS), Princeton, New Jersey, USA, March 2014.

[21] M. Chiang, Geometric programming for communication systems Found. Trends Commun. Inf. Theory 2 (July (1-2)) (2005) 1-154.

[22] M. Krunz, A. Muqattash, S. Lee, Transmission power control in wireless ad hoc networks: challenges, solutions, and open issues, IEEE Netw. 18 (October (5)) (2004) 8-14.

[23] M. Wiczanowski, S. Stanczak, H. Boche, Performance and interference control in wireless ad-hoc and mesh networks - a generalized Lagrangian approach, IEEE Trans. Signal Process. 56 (August (8)) (2008) 4039-4052.

[24] N. Gatsis, G. Giannakis, Power control with imperfect exchanges and applications to spectrum sharing, IEEE Trans. Signal Process. 59 (July (7)) (2011) 3410-3423.

[25] V. Douros, G. Polyzos, Review of some fundamental approaches for power control in wireless networks, Elsevier Comput. Commun. 34 (13) (2011) 1580-1592.

[26] M. Hong, Z.Q. Luo, Signal processing and optimal resource allocation for the interference channel, EURASIP E-Reference Signal Processing, http://arxiv.org, 2012.

[27] A. Ghasemi, E. Sousa, Fundamental limits of spectrum-sharing in fading environments, IEEE Trans. Wireless Commun. 6 (February (2)) (2007) 649-658

[28] L. Le, E. Hossain, Resource allocation for spectrum underlay in cognitive radio networks, IEEE Trans. Wirel. Commun. 7 (December (12)) (2008) 5306-5315.

[29] X. Kang, Y. Liang, A. Nallanathan, R. Zhang, Optimal power allocation for fading channels in cognitive radio networks: ergodic capacity and outage capacity, IEEE Trans. Wireless Commun. 8 (February (2)) (2009) 940-950.

[30] M. Charafeddine, A. Sezgin, A. Paulraj, Rate region frontiers for $n-$ user interference channel with interference as noise, in: Allerton Conference on Communication, Control, and Computing, Monticell Illinois, USA, September 2007.

[31] M. Charafeddine, A. Paulraj, Maximum sum rates via analysis of 2user interference channel achievable rates region, in: Annual Conference on Information Sciences and Systems (CISS), Baltimore, MD, USA, March 2009

[32] C. Yang, J. Li, Z. Tian, Optimal power control for cognitive radio networks under coupled interference constraints: a cooperative game-theoretic perspective, IEEE Trans. Veh. Technol. 39 (May (4)) (2010) 1696-1706.

[33] J. Rajaselharan, V. Koivunen, Cooperative game-theoretic approach to spectrum sharing in cognitive radios, Signal Process. January (106) (2015) 15-29.

[34] L. Li, F. Khan, M. Pesavento, T. Ratnarajah, S. Prakriya, Sequential search based power allocation and beamforming design in overlay cognitive radio, Signal Process. April (97) (2014) 221-231.

[35] F. Shen, E. Jorswieck, Universal non-linear cheat-proof pricing framework for wireless multiple access channels, IEEE Trans. Wirel Commun. 13 (March (3)) (2014) 1436-1448.

[36] V. Douros, G. Polyzos, S. Toumpis, Negotiation-based distributed power control in wireless networks with autonomous nodes, in: IEEE Vehicular Technology Conference (VTC-Spring), Budapest, Hungary.

[37] E. Panaousis, C. Politis, G. Polyzos, Maximizing network throughput: power control using game theory in a shared open spectrum, IEEE Veh. Technol. Mag. 4 (September (3)) (2009) 33-39.

[38] C. Shi, R. Berry, M. Honig, Monotonic convergence of distributed interference pricing in wireless networks, in: IEEE International Symposium on Information Theory (ISIT), Seoul, South Korea, June 2009.

[39] S. Kim, G. Giannakis, Optimal resource allocation for MIMO ad hoc cognitive radio networks, IEEE Trans. Inf. Theory 57 (May (5)) (2011) 3117-3131.

[40] F. Shen, Resource allocation for multiple access and broadcast channels under quality of service requirements based on strategy proof pricing (Ph.D. thesis), Technische Universität Dresden, Germany, November 2014.

[41] M. Gastpar, On capacity under receive and spatial spectrum-sharing constraints, IEEE Trans. Inf. Theory 53 (January (2)) (2007) 471-487. 
[42] A. Behbahani, A. Eltawil, Amplify-and-forward relay networks under received power constraint, IEEE Trans. Wirel. Commun. 8 (November (11)) (2009) 5422-5426.

[43] A. Pérez-Neira, M. Lagunas, M. Vázquez, Autonomous design for the MISO interference channel with mask constraints at reception, in: IEEE Applied Electromagnetics Conference (AEMC), Kolkata, India, December 2011.

[44] M. Hong, A. Garcia, Equilibrium pricing of interference in cognitive radio networks, IEEE Trans. Signal Process. 59 (August (12)) (2011) 6058-6072.

[45] Y. Su, M. van der Schaar, Structural solutions for additively coupled sum constrained games, IEEE Trans. Commun. 60 (December (12)) (2012) 3779-3796.

[46] N. Hassan, C. Yuen, S. Saeed, Z. Zhang, Power control for sum rate maximization on interference channels under sum power constraint, IEEE Trans. Veh. Technol. 64 (February (2)) (2015) 593-609.

[47] M. Ehrgott, Multicriteria Optimization, Springer-Verlag, Berlin, Germany, 2005.

[48] E. Bjornson, E. Jorswieck, M. Debbah, B. Ottersten, Multiobjective signal processing optimization: the way to balance conflicting metrics in 5G systems, IEEE Signal Process. Mag. 31 (November (6)) (2014) 14-23.

[49] E. Bjornson, E. Jorswieck, M. Debbah, B. Ottersten, Multiobjective signal processing optimization: the way to balance conflicting metrics in 5G systems, IEEE Signal Process. Mag. 31 (6) (2014) 14-23, http://dx.doi.org/10.1109/MSP.2014.2330661. ISSN 1053-5888.

[50] Y. Sawaragi, H. Nakayama, T. Tanino, Theory of Multiobjective Optimization, Academic Press, Oxford, UK, 1985.

[51] I. Stancu-Minasian, Fractional programming: theory, Methods and Applications, Kluwer Academic Publishers, Dordrecht, The Netherlands, 1997.
[52] M. Charafeddine, A. Sezgin, Z. Han, A. Paulraj, Achievable and crystallized rate regions of the interference channel with interference as noise, IEEE Trans. Wirel. Commun. 11 (3) (2012) 1100-1111, http: //dx.doi.org/10.1109/TWC.2012.010312.110497. ISSN 1536-1276.

[53] S. Elaydi, An Introduction to Difference Equations, 3rd ed. Springer Science Business Media, USA, 2005.

[54] S. Grandhi, J. Zander, Constrained power control in cellular radio systems, in: 1994 IEEE 44th Vehicular Technology Conference, vol.2, pp. 824-828, ISSN 1090-3038, http://dx.doi.org/10.1109/VETEC.1994. 345205, 1994.

[55] M. Kaykobad, Positive solutions of positive linear systems, Linear Algebra Appl. 64 (January (2)) (1985) 133-140.

[56] M. Bengtsson, B. Ottersten, Optimal and suboptimal transmit beamforming, Handbook of Antennas in Wireless Communications, CRC Press, 2011.

[57] D. Gerlach, A. Paulraj, Base station transmitting antenna arrays for multipath environments, Signal Process. 54 (October) (1996) 59-73.

[58] R. Mochaourab, E. Jorswieck, Robust beamforming in interference channels with imperfect transmitter channel information, Signal Process. 92 (10) (2012) 2509-2518.

[59] C. Zou, T. Jin, C. Chigan, Z. Tian, QoS-aware distributed spectrum sharing for heterogeneous wireless cognitive network, Comput. Netw. 52 (March (4)) (2008) 864-878.

[60] W. Dinkelbach, On nonlinear fractional programming, Manag. Sci. 13 (7) (1967) 492-498, http://dx.doi.org/10.2307/2627691. ISSN 00251909. 